\title{
Clinical comparison of low-volume agents (oral sulfate solution and sodium picosulfate with magnesium citrate) for bowel preparation: the EASE study
}

Jeeyeon Kim ${ }^{1}$, Hyun Gun Kim ${ }^{1}$, Kyeong Ok Kim², Hyung Wook Kim³, Jongha Park ${ }^{4}$, Jeong-Sik Byeon ${ }^{5}$, Sung-Wook Hwang ${ }^{5}$, Hyun Deok Shin ${ }^{6}$, Jeong Eun Shin ${ }^{6}$, Hyo-Joon Yang ${ }^{7}$, Hyun Seok Lee ${ }^{8}$, Yunho Jung ${ }^{9}$, Young-Seok Cho ${ }^{10}$, Young Eun Joo ${ }^{11}$, Dae-Seong Myung ${ }^{11}$, Kyu Chan Huh ${ }^{12}$, Eu Mi Ahn ${ }^{13}$

${ }^{1}$ Department of Internal Medicine, Soonchunhyang University College of Medicine, Seoul; ${ }^{2}$ Department of Internal Medicine, Yeungnam University College of Medicine, Daegu; ${ }^{3}$ Department of Internal Medicine, Pusan National University College of Medicine, Busan; ${ }^{4}$ Department of Internal Medicine, Inje University College of Medicine, Busan; ${ }^{5}$ Department of Gastroenterology, University of Ulsan College of Medicine, Seoul; ${ }^{6}$ Department of Internal Medicine, Dankook University College of Medicine, Cheonan; ${ }^{7}$ Department of Internal Medicine, Sungkyunkwan University School of Medicine, Seoul; ${ }^{8}$ Department of Internal Medicine, School of Medicine, Kyungpook National University, Daegu; ${ }^{9}$ Department of Internal Medicine, Soonchunhyang University College of Medicine, Cheonan; ${ }^{10}$ Department of Internal Medicine, College of Medicine, The Catholic University of Korea, Seoul; ${ }^{11}$ Department of Internal Medicine, Chonnam National University Medical School, Gwangju; ${ }^{12}$ Department of Internal Medicine, Konyang University College of Medicine, Daejeon, ${ }^{13}$ Digestive Disease Center, Soonchunhyang University Hospital, Seoul, Korea

Background/Aims: This study compared the efficacy, compliance, and safety of bowel preparation between sodium picosulfate with magnesium citrate (SPMC) and oral sulfate solution (OSS). Methods: A prospective randomized multicenter study was performed. Split preparation methods were performed in both groups; the SPMC group, 2 sachets on the day before, and 1 sachet on the day of the procedure, the OSS group, half of the OSS with $1 \mathrm{~L}$ of water on both the day before and the day of the procedure. The adenoma detection rate (ADR), adequacy of bowel preparation using the Boston Bowel Preparation Scale (BBPS) score, patient satisfaction on a visual analog scale (VAS), and safety were compared between the 2 groups. Results: This study analyzed 229 patients (121 in the SPMC group and 108 in the OSS group). ADR showed no differences between 2 groups (51.7\% vs. $41.7 \%, P>0.05)$. The mean total BBPS score (7.95 vs. 8.11, $P>0.05)$ and adequate bowel preparation rate (94.9\% vs. $96.3 \%, P>0.05)$ were similar between the 2 groups. The mean VAS score for taste ( $7.62 \mathrm{vs.} 6.87, P=0.006)$ was significantly higher in the SPMC group than in the OSS group. There were no significant differences in any other safety variables between the 2 groups except nausea symptom ( $36.1 \%$ vs. $20.3 \%, P=0.008)$. Conclusions: Bowel preparation for colonoscopy using low volume OSS and SPMC yielded similar ADRs and levels of efficacy. SPMC had higher levels of satisfaction for taste and feeling than did OSS. (Intest Res 2019;17:413-418)

Key Words: Colonoscopy; Bowel preparation; Oral sulfate solution; Sodium picosulfate with magnesium citrate

\section{INTRODUCTION}

Colonoscopy is the most sensitive diagnostic tool for colon

Received December 6, 2018. Revised January 28, 2019

Accepted February 20, 2019.

Correspondence to Hyun Gun Kim, Department of Internal Medicine,

Soonchunhyang University Hospital, 59 Daesagwan-ro, Yongsan-gu,

Seoul 04401, Korea. Tel: +82-2-710-3072, Fax: +82-2-709-9868, E-mail:

medgun@schmc.ac.kr

ORCID Hyun Gun Kim (https://orcid.org/0000-0001-7545-4638) cancer. ${ }^{1}$ However, a recent meta-analysis reported that more than $20 \%$ of polyps are overlooked during colonoscopy. The factors that influence the polyp miss rate are colonoscopy quality management, including the clinician's ability to perform cecum insertion; colonoscope withdrawal time; and the adequacy of bowel preparation. ${ }^{3}$ The degree of patient compliance with the bowel cleansing regimen and the efficacy of the bowel cleansing agent may impact the adequacy of bowel preparation. Therefore, both the laxative used and the cooper- 
ation of the patient are important.

The most commonly used laxative is polyethylene glycol (PEG) solution, which does not cause dehydration or electrolyte imbalance, and has a good cleansing effect. However, the poor taste of PEG solutions hampers consumption of the large volume typically required for bowel preparation. ${ }^{4}$ To overcome this issue, oral sulfate solution (OSS), sodium picosulfate with magnesium citrate (SPMC), and PEG with ascorbic acid (PEG plus Asc $)^{5}$ have been developed and are now commercially available.

SPMC does not contain phosphoric acid, which can cause renal dysfunction. Unlike PEG, which must be taken with at least $2 \mathrm{~L}$ of water, 2 sachets of SPMC (PicoLite ${ }^{\circledR}$ ) are taken with $150 \mathrm{~mL}$ of water, followed by $250 \mathrm{~mL}$ of water per hour until diarrhea stops. Therefore, a relatively small dose of SPMC is thought to be effective. ${ }^{6,7}$

OSS was developed to overcome the nephrotoxicity and electrolytic imbalance caused by sodium phosphate preparations, and has been proven to be safe and not absorbed by the body. ${ }^{8}$ According to studies in Western countries, the success rate of bowel preparation using OSS is similar or higher than that of PEG, ${ }^{9}$ and OSS showed a higher ADR and superior safety compared to SPMC. ${ }^{10}$

Direct comparison studies of low-dose laxatives are scarce, ${ }^{11}$ and direct comparison studies of SPMC and OSS are even more so. Therefore, in this study, we directly compared the adequacy and safety of bowel preparation for colonoscopy between OSS and SPMC.

\section{METHODS}

\section{Study Design and Methods}

A prospective, randomized, single-blinded study, multicenter (11 centers nationwide) trial was performed. The institutional review board of each center approved the study, which was registered at the open registry of the Clinical Research Information Service (http://cris.nih.go.kr) under the identifier KCT00 03148. After informed consent, the patients were randomly assigned to the OSS or SPMC group using randomization software.

The inclusion criteria were outpatients undergoing their first colonoscopy or an at-least 5 year interval since the last colonoscopy, age of 50 to 75 years, and provision of written informed consent. Patients aged less than 50 or more than 75 years of age, and those with bowel obstruction, severe constipation (fewer than 3 bowel movements per week, or regular or inter- mittent laxative use), previous bowel surgery, cirrhosis, ascites, chronic kidney disease (serum creatinine level $>3 \mathrm{mg} / \mathrm{dL}$ for $>6$ months), heart failure, heart disease (ischemic heart disease or coronary artery disease within the last 6 months), or pregnancy were excluded from the study. Patients who refused to sign the consent form were also excluded.

\section{Preparation Instructions}

Patients were given detailed verbal and written bowel preparation instructions before colonoscopy. From 3 days pre-procedure, intake of food containing seeds, fruits, mushrooms, seaweeds, etc., which could interfere with the endoscopy, was restricted. Patients undergoing colonoscopy in the morning consumed a liquid diet on the day before the procedure, and had dinner before $6 \mathrm{PM}$.

The patients in the OSS (Suclear ${ }^{\circledR}$; Pharmbio Korea Inc., Seoul, Korea) group were instructed to start the preparation at 7 PM and to use a split-dose method, in which $177 \mathrm{~mL}$ of OSS was mixed with $323 \mathrm{~mL}$ water, followed 1 hour later by $1 \mathrm{~L}$ of water was taken at $7 \mathrm{PM}$ the day before the colonoscopy and another of the same preparation was performed done 3 to 6 hours before the colonoscopy. We used a pre-mixed oral solution of SPMC (Picosolution ${ }^{\circledR}$; Pharmbio Korea Inc.), which promotes patient compliance by obviating the need to mix the powder with water prior to taking it. The patients in the SPMC group were instructed to drink 3 bottles of SPMC; drink each 1 bottle of SPMC ( $170 \mathrm{~mL})$ at 6 and $8 \mathrm{PM}$, and each followed 1 hour later by $1 \mathrm{~L}$ of water taken on the day before the procedure. For morning endoscopies, the patients took another bottle of SPMC with $1 \mathrm{~L}$ of water 3 to 6 hours before the colonoscopy.

\section{Assessments}

\section{1) Efficacy and Safety}

The colonoscopy procedures and assessments of bowel preparation were performed at the participating centers. Efficacy was evaluated by assessing the quality of colonoscopy and bowel cleansing. Colonoscopy quality was assessed by comparing the adenoma detection rate (ADR) between the 2 groups. The colonoscope withdrawal time was recorded and the adequacy of bowel preparation in the right, transverse, and left colon was assessed using the Boston Bowel Preparation Scale (BBPS), a validated tool for the evaluation of bowel integrity. ${ }^{12}$ Adequate bowel preparation was defined as a score of $\geq 2$ points in all areas, and excellent bowel preparation as a score of 7 to 9 points in all areas.

Compliance with the laxative regimen was assessed by de- 
termining the proportion of patients who did not take the laxative as directed, measured on a visual analog scale (VAS) from 0 (very bad) to 10 (very good). Patient satisfaction was also assessed by VAS scores, for feeling, taste, and dose of the laxative, and by recording any symptoms experienced after taking it.

Safety was assessed based on the incidence of adverse events reported as yes or no in the questionnaire immediately before the colonoscopy. A questionnaire was administered to assess nausea, vomiting, abdominal pain, abdominal distension, dry mouth, dizziness, headache, or paresthesia during consumption of the laxatives.

We also evaluated the electrolyte levels and neurological parameters of the patients using blood samples obtained immediately prior to colonoscopy. Changes in the colonic mucosa and the presence of aphthous ulcer were also recorded.

\section{2) Statistical Analysis}

We hypothesized that $90 \%$ of the patients in the OSS and SPMC groups would show adequate bowel preparation. The null hypothesis was rejected if the rate of adequate bowel preparation in the SPMC and OSS groups differed by $>10 \%$. Assuming a dropout rate of $10 \%$ and based on a statistical power of $0.2,124$ patients were required per group, for a total of $248 \mathrm{pa}-$ tients.

We performed per protocol analyses using SPSS software version 20.0 (IBM Corp., Armonk, NY, USA). Numerical variables were compared by $t$-test and categorical variables by chisquare test. $P$-values $<0.05$ were considered indicative of statistical significance.

\section{RESULTS}

\section{Patients}

A total of 251 patients was enrolled in the study, of whom 22 were excluded due to withdrawing consent $(n=4)$ or protocol violation $(\mathrm{n}=18)$. Thus, the ITT population consisted of 108 patients of the OSS group and 121 patients of the SPMC group (Fig. 1). Of these 229 patients, three patients in the SPMC group were withdrawn from the study due to a change in schedule or cancellation, 226 patients (108 in the OSS group and 118 in the SPMC group) completed the entire preparation process and were analyzed as the PP population, ultimately. There were no significant differences in mean height, weight, or body mass index between the 2 groups (Table 1 ).

\section{Efficacy}

The ADR $(41.7 \%$ vs. $51.7 \%, P=0.131)$ and colonoscope withdrawal time (10.43 minutes vs. 10.24 minutes, $P=0.774$ ) were not different between the 2 groups by PP analysis (Table 2).

The total BBPS score was 8.11 points in the OSS group and 7.95 points in the SPMC group $(P=0.343)$. The adequate bowel preparation rate was $96.3 \%$ in the OSS group and $94.9 \%$ in the SPMC group $(P=0.614)$. The excellent bowel preparation rate was $82.4 \%$ in the OSS group and $81.4 \%$ in the SPMC group. The OSS group had a significantly higher BBPS score for the transverse colon compared to the SPMC group (2.79 vs. 2.66, respectively; $P=0.046)$. The BBPS scores for the ascending and descending colon were not different between the 2 groups.

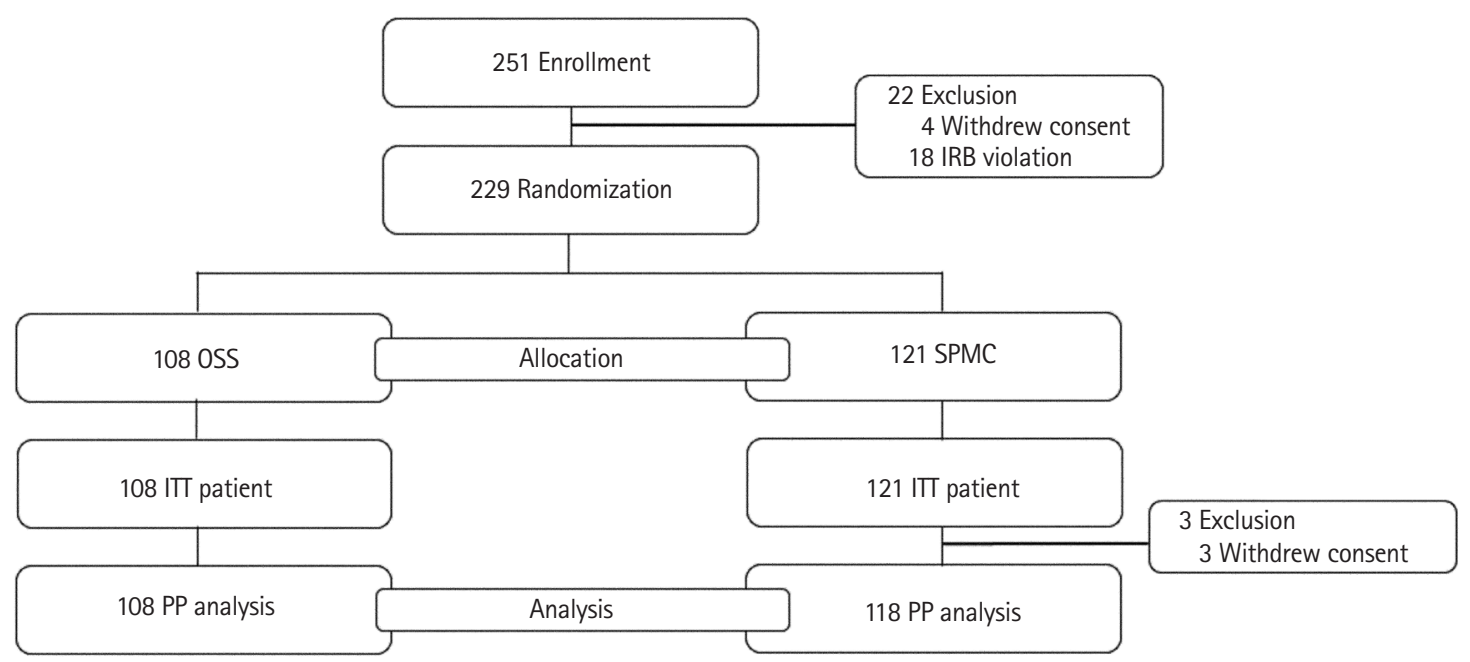

Fig. 1. Characteristics of the study participants. IRB, institutional review board; OSS, oral sulfate solution; IT, intention-to-treat; PP, per protocol; SPMC, sodium picosulfate with magnesium citrate. 
Table 1. Demographic Characteristics of the Intention-to-Treat Population

\begin{tabular}{lccc}
\hline Characteristic & OSS $(\mathrm{n}=108)$ & SPMC $(\mathrm{n}=121)$ & $P$-value \\
\hline Sex (male/female) & $46(42.6) / 62(57.4)$ & $48(39.7) / 73(60.3)$ & 0.654 \\
Age $(\mathrm{yr})$ & 60.9 & 59.8 & 0.247 \\
Height $(\mathrm{m})$ & 1.61 & 1.61 & 0.960 \\
Weight $(\mathrm{kg})$ & 61.98 & 62.03 & 0.967 \\
BMI $\left(\mathrm{kg} / \mathrm{m}^{2}\right)$ & 23.68 & 23.73 & 0.889 \\
\hline
\end{tabular}

Values are presented as number (\%) or mean.

OSS, oral sulfate solution; SPMC, sodium picosulfate with magnesium citrate.

Table 2. Bowel Cleansing Efficacy in the OSS and SPMC Groups

\begin{tabular}{lrrr}
\hline & $\begin{array}{c}\text { OSS } \\
(\mathrm{n}=108)\end{array}$ & $\begin{array}{c}\text { SPMC } \\
(\mathrm{n}=118)\end{array}$ & P-value \\
\hline Adenoma detection rate & $45(41.7)$ & $61(51.7)$ & 0.131 \\
Withdrawal time (min) & $10.43 \pm 5.13$ & $10.24 \pm 4.63$ & 0.774 \\
Bowel preparation & & & \\
$\quad$ BBPS (right colon) & $2.60 \pm 0.58$ & $2.58 \pm 0.58$ & 0.740 \\
BBPS (transverse colon) & $2.79 \pm 0.43$ & $2.66 \pm 0.51$ & 0.046 \\
BBPS (left colon) & $2.72 \pm 0.45$ & $2.72 \pm 0.47$ & 0.976 \\
BBPS (total) & $8.11 \pm 1.19$ & $7.95 \pm 1.36$ & 0.343 \\
Adequate bowel preparation & $104(96.3)$ & $112(94.9)$ & 0.614 \\
Excellent bowel preparation & $89(82.4)$ & $96(81.4)$ & 0.838 \\
\hline
\end{tabular}

Values are presented as number (\%) or mean \pm SD.

OSS, oral sulfate solution; SPMC, sodium picosulfate with magnesium citrate; BBPS, Boston Bowel Preparation Scale.

\section{Patient Satisfaction}

In the OSS and SPMC groups, $91.7 \%$ and $92.4 \%$ of the patients, respectively, indicated that they were willing to undergo a repeat colonoscopy using the same laxative $(P=0.845)$.

We assessed the patients' level of satisfaction with the laxatives based on their VAS scores for feeling, taste, and dose. The feeling VAS score was 7.63 in the SPMC group and 7.09 in the OSS group $(P=0.035)$. The taste VAS score was 7.62 in the SPMC group and 6.87 in the OSS group $(P=0.006)$. There were no differences between the 2 groups in the VAS score for laxative dose by PP analysis (Table 3 ).

\section{Safety}

The most common complaints after taking the laxatives were abdominal bloating, nausea, abdominal pain, and oral symptoms. Thirty-eight patients (35.2\%) in the OSS group and 34 $(28.8 \%)$ in the SPMC group complained of abdominal bloat-
Table 3. Patient Satisfaction in the OSS and SPMC Groups

\begin{tabular}{lccc}
\hline & OSS $(\mathrm{n}=108)$ & SPMC $(\mathrm{n}=118)$ & $P$-value \\
\hline Taste & 6.87 & 7.62 & 0.006 \\
Feeling & 7.09 & 7.63 & 0.035 \\
Volume & 7.31 & 7.57 & 0.363 \\
\hline
\end{tabular}

Values are presented as mean of visual analog scale score. OSS, oral sulfate solution; SPMC, sodium picosulfate with magnesium citrate.

Table 4. Adverse Events in the OSS and SPMC Groups

\begin{tabular}{lccc}
\hline Adverse event & OSS $(n=108)$ & SPMC $(n=118)$ & $P$-value \\
\hline Nausea & $39(36.1)$ & $24(20.3)$ & 0.008 \\
Vomiting & $9(8.3)$ & $4(3.4)$ & 0.178 \\
Abdominal pain & $18(16.7)$ & $12(10.2)$ & 0.150 \\
Abdominal distention & $38(35.2)$ & $34(28.8)$ & 0.304 \\
Dry mouth & $14(13.0)$ & $13(11.0)$ & 0.652 \\
Dizziness & $9(8.3)$ & $11(9.3)$ & 0.794 \\
Sensory disorder & $2(1.9)$ & $4(3.4)$ & 0.473 \\
Numbness & 0 & $1(0.8)$ & 0.338 \\
Mucosal change & 0 & $1(0.8)$ & 0.338 \\
Convulsion & 0 & 0 & - \\
General weakness & $5(4.6)$ & $5(4.2)$ & 0.886 \\
Mental change & 0 & 0 & - \\
\hline
\end{tabular}

Values are presented as number (\%).

OSS, oral sulfate solution; SPMC, sodium picosulfate with magnesium citrate.

ing $(P>0.05)$. Thirty-nine patients $(36.1 \%)$ in the OSS group and $24(20.3 \%)$ in the SPMC group reported experiencing nausea $(P=0.008)$.

Other complaints were abdominal pain ( $16.7 \%$ vs. $10.2 \%$ for the OSS vs. SPMC groups), vomiting ( $8.3 \%$ vs. $3.4 \%)$, dry mouth (13.0\% vs. $11.0 \%$ ), dizziness ( $8.3 \%$ vs. $9.3 \%$ ), sensory disorder (1.9\% vs. $3.4 \%$ ), generalized weakness ( $4.6 \%$ vs. $4.2 \%$ ), and numbness ( $0 \%$ vs. $0.8 \%$ ) (Table 4$)$.

A change in the mucosa or aphthous ulceration after colonoscopy was reported by only 1 patient, who was in the SPMC group. There were no significant differences in the values of the other laboratory parameters between the 2 groups (Table 5 ).

\section{DISCUSSION}

Lower-dose laxatives are widely used for colonoscopy. Bowel preparation using OSS is adequate and safe. However, the cleansing effect of the SPMC method remains unclear. To im- 
Table 5. Laboratory Results of the OSS and the SPMC Groups

\begin{tabular}{lccc}
\hline & $\begin{array}{c}\text { OSS } \\
(\mathrm{n}=108)\end{array}$ & $\begin{array}{c}\text { SPMC } \\
(\mathrm{n}=118)\end{array}$ & P-value \\
\hline Sodium (Na) & 140.73 & 141.34 & \\
Hyponatremia & $1(0.9)$ & $5(4.2)$ & 0.203 \\
Hypernatremia & $1(0.9)$ & $2(1.7)$ & 0.254 \\
Potassium (K) & 4.18 & 4.04 & 0.008 \\
Hyperkalemia & $1(0.9)$ & $1(0.8)$ & 0.957 \\
Chloride (Cl) & 102.55 & 101.44 & \\
Hyperchloridemia & $3(2.8)$ & $3(2.5)$ & 0.006 \\
Hypochloridemia & $1(0.9)$ & 0 & 0.585 \\
Magnesium (Mg) & 2.25 & 2.32 & 0.089 \\
Hypermagnesemia & $5(4.6)$ & $8(6.8)$ & 0.473 \\
Phosphate (P) & 3.52 & 3.54 & \\
Hypophosphatemia & $1(0.9)$ & 0 & 0.845 \\
Hyperphosphatemia & $2(1.9)$ & $2(1.7)$ & 0.579 \\
Calcium (Ca) & 9.19 & 9.15 & 0.479 \\
Hypocalcemia & $11(10.2)$ & $10(8.5)$ & 0.677 \\
BUN & 13.31 & 11.60 & 0.023 \\
Cr & 0.83 & 0.79 & 0.158 \\
eGFR & 86.53 & 89.06 & 0.274 \\
\hline
\end{tabular}

Values are presented as mean or number (\%).

OSS, oral sulfate solution; SPMC, sodium picosulfate with magnesium citrate; $\mathrm{Cr}$, creatinine; eGFR, estimated glomerular filtration rate.

prove the cleansing effect of SPMC, some researchers have proposed a split method involving 2 sachets taken the evening before the day of the colonoscopy and 4 to 5 hours before the colonoscopy combined with a laxative. ${ }^{13,14}$ SPMC showed a variable cleansing efficacy according to dosage (2 sachets vs. 3 sachets) or split-dose method (same-day split dose vs. 2-day split dose). In a recent study of OSS versus 2 sachets of SPMC, the rates of excellent or good bowel preparation were $94.7 \%$ and $85.7 \%$, respectively, according to the American Society for Gastrointestinal Endoscopy colon cleansing score $(P=0.006){ }^{10}$ A recent Korean study compared the efficacy of PEG plus ascorbic acid with a split-dose regimen comprising 3 sachets of SPMC. ${ }^{7}$ As results, 3 sachets of SPMC produced a high level of satisfaction in preparation quality and ADR.

Few studies have directly compared the low-dose laxatives OSS and SPMC The present study compared OSS and 3 sachets of SPMC based on the excellent result of previous study. ${ }^{7}$ In this study, the ADR was satisfactory and was not different between the 2 groups $(P>0.05)$. In addition, the bowel preparation quality of the 2 groups was similar, although BBPS of the transverse colon was higher in the OSS group than the SPMC group. The adequate bowel preparation rate all excellent in both groups; $96.3 \%$ in the OSS group and $94.9 \%$ in the SPMC group $(P>0.05)$. The excellent bowel preparation rate (BBPS score, $7-9$ points) was $82.4 \%$ in the OSS group and $81.4 \%$ in the SPMC group $(P>0.05)$. Therefore, OSS and 3 sachets of SPMC were similarly effective for bowel preparation pre colonoscopy.

The feeling VAS score after taking the laxatives, as well as the taste VAS score, were significantly higher in the SPMC group than in the OSS group. There were no significant differences between the 2 groups in the VAS score for laxative dose. The overall satisfaction rate was higher in the SPMC group than in the OSS group. Among the patients in the OSS and SPMC groups, $91.7 \%$ and $92.4 \%$, respectively, were willing to undergo a repeat colonoscopy using the same laxative.

Both groups had similar overall rates of adverse events related to bowel preparation. However, the overall rate of side effects was higher in the OSS group; in particular, only the frequency of nausea differed significantly between the 2 groups. Moreover, there were no significant differences between the 2 groups in the levels of electrolytes and blood urea nitrogen/creatinine.

A limitation of this study was the safety concern regarding consumption of low-volume purgatives. Nevertheless, we have limited evidence on low-volume purgatives for patients with comorbidities, so we excluded chronic patients with cardiovascular disease or renal insufficiency. Our results may not be generalizable or applicable to these high-risk patients. Another limitation is that the current study had single blinded due to differences in the administration of the bowel preparations, which may be a source of bias, although precautions were taken to ensure that the endoscopists remained blinded to the bowel preparation method.

In conclusion, 2 low-volume purgatives, OSS and SPMC, were satisfactory in terms of the ADR or the quality of bowel cleansing for colonoscopy. Although SPMC showed superior satisfaction regarding taste and feeling, both agents resulted in more qualified colonoscopies with improved compliance.

\section{FINANCIAL SUPPORT}

The authors received no financial support for the research, authorship, and/or publication of this article

\section{CONFLICT OF INTEREST}

No potential conflict of interest relevant to this article was reported. 


\section{AUTHOR CONTRIBUTION}

Conceptualization: Kim HG. Methodology: Kim HG. Formal analysis: Kim HG, Kim J. Project administration: Kim HG, Ahn EM, Huh KC. Writing - original draft: Kim J, Kim HG. Writing review and editing: Kim KO, Kim HW, Park J, Byeon JS, Hwang SW, Shin HD, Shin JE, Yang HJ, Lee HS, Jung Y, Cho YS, Myung DS, Joo YE. Approval of final manuscript: all authors.

\section{REFERENCES}

1. Graser A, Stieber P, Nagel D, et al. Comparison of CT colonography, colonoscopy, sigmoidoscopy and faecal occult blood tests for the detection of advanced adenoma in an average risk population. Gut 2009;58:241-248.

2. van Rijn JC, Reitsma JB, Stoker J, Bossuyt PM, van Deventer SJ, Dekker E. Polyp miss rate determined by tandem colonoscopy: a systematic review. Am J Gastroenterol 2006;101:343-350.

3. Rex DK, Petrini JL, Baron TH, et al. Quality indicators for colonoscopy. Am J Gastroenterol 2006;101:873-885.

4. Parra-Blanco A, Ruiz A, Alvarez-Lobos M, et al. Achieving the best bowel preparation for colonoscopy. World J Gastroenterol 2014;20:17709-17726.

5. Ell C, Fischbach W, Bronisch HJ, et al. Randomized trial of low-volume PEG solution versus standard PEG + electrolytes for bowel cleansing before colonoscopy. Am J Gastroenterol 2008;103:883-893.

6. Lim JS, Choi DS, Kim YJ, et al. Characterization of Escherichia coli-producing extended-spectrum beta-lactamase (ESBL) isolated from chicken slaughterhouses in South Korea. Foodborne Pathog Dis 2015;12:741-748.

7. Jeon SR, Kim HG, Lee JS, et al. Randomized controlled trial of low-volume bowel preparation agents for colonic bowel prep- aration: 2-L polyethylene glycol with ascorbic acid versus sodium picosulfate with magnesium citrate. Int J Colorectal Dis 2015;30:251-258.

8. Di Palma JA, Rodriguez R, McGowan J, Cleveland Mv. A randomized clinical study evaluating the safety and efficacy of a new, reduced-volume, oral sulfate colon-cleansing preparation for colonoscopy. Am J Gastroenterol 2009;104:2275-2284.

9. Rex DK, Di Palma JA, Rodriguez R, McGowan J, Cleveland M. A randomized clinical study comparing reduced-volume oral sulfate solution with standard 4-liter sulfate-free electrolyte lavage solution as preparation for colonoscopy. Gastrointest Endosc 2010;72:328-336.

10. Rex DK, DiPalma JA, McGowan J, Cleveland M. A comparison of oral sulfate solution with sodium picosulfate: magnesium citrate in split doses as bowel preparation for colonoscopy. Gastrointest Endosc 2014;80:1113-1123.

11. Yoshioka K, Connolly AB, Ogunbiyi OA, Hasegawa H, Morton DG, Keighley MR. Randomized trial of oral sodium phosphate compared with oral sodium picosulphate (Picolax) for elective colorectal surgery and colonoscopy. Dig Surg 2000;17:6670.

12. Lai EJ, Calderwood AH, Doros G, Fix OK, Jacobson BC. The Boston Bowel Preparation Scale: a valid and reliable instrument for colonoscopy-oriented research. Gastrointest Endosc 2009;69:620-625.

13. Flemming JA, Vanner SJ, Hookey LC. Split-dose picosulfate, magnesium oxide, and citric acid solution markedly enhances colon cleansing before colonoscopy: a randomized, controlled trial. Gastrointest Endosc 2012;75:537-544.

14. Hookey LC, Vanner SJ. Pico-salax plus two-day bisacodyl is superior to pico-salax alone or oral sodium phosphate for colon cleansing before colonoscopy. Am J Gastroenterol 2009; 104:703-709. 